\title{
La Réception de la sociologie américaine en France
} 1945-1960

Jean-Christophe Marcel

\section{(2) OpenEdition}

\section{Journals}

Édition électronique

URL : http://journals.openedition.org/ress/1044

DOI : $10.4000 /$ ress. 1044

ISSN : 1663-4446

Éditeur

Librairie Droz

\section{Édition imprimée}

Date de publication : 15 décembre 2011

Pagination : 197-230

ISBN : 978-2-600-01575-2

ISSN : 0048-8046

\section{Référence électronique}

Jean-Christophe Marcel, «La Réception de la sociologie américaine en France », Revue européenne des sciences sociales [En ligne], 49-2 | 2011, mis en ligne le 01 janvier 2015, consulté le 19 avril 2019. URL: http://journals.openedition.org/ress/1044; DOI : 10.4000/ress.1044 


\title{
LA RÉCEPTION DE LA SOCIOLOGIE AMÉRICAINE EN FRANCE
}

1945-1960

\author{
JEAN-CHRISTOPHE MARCEL \\ Université Paris-Sorbonne - GEMASS \\ Jean-Christophe.Marcel@paris-sorbonne.fr
}

\begin{abstract}
Résumé. Cet article étudie la réception de la sociologie américaine en France à partir d'un corpus d'articles et comptes rendus tirés de L'Année Sociologique et des Cahiers Internationaux de Sociologie sur la période 1945-1959. À rebours d'une tradition constructiviste tenace, en France du moins, il essaie de montrer que cette réception ne se limita pas à la substitution d'une sociologie abstraite et théorique par une sociologie dite de terrain, grâce à l'introduction des méthodes américaines d'investigation empirique de terrain. Elle manifesta également l'ambition théorique de combler les lacunes de l'approche sociologique des «petits groupes» au moyen d'un appareillage conceptuel soucieux de donner un sens aux faits observés en les rapportant à la «totalité» de la société. Ceci conduit à remettre en cause l'idée d'une totale «refondation» de la sociologie française après 1945.
\end{abstract}

Mots-clés: réception, sociologie américaine, histoire de la sociologie française, société, petits groupes

\begin{abstract}
This article is a study of how French sociologists used to read American sociology after World War II. The study of a corpus of texts published in both French journals L'Année Sociologique and the Cahiers Internationaux de Sociologie from 1945 to 1959, shows that the so-called rebuilding of French Sociology was not the change from a theoretical analysis inspired by philosophical questions, to an empirical enquiry inspired by the Americans, usually described by historians of French sociology. Against a strong contructivist tradition, it aims at proving how the content of those articles and reviews proves that American "small groups" studies and their methods were read in a special way: to observe and understand social facts only if they are linked with the idea of society still viewed as a whole totality.
\end{abstract}

Keywords: reception, American sociology, history of French sociology, society, small groups 


\section{INTRODUCTION}

Dans l'histoire de la discipline sociologique en France, la référence à son homologue américaine tient une place à part. Elle aurait impulsé une «refondation» de la sociologie française (Chapoulie, I99I), qu'on tient d'ordinaire pour significative du passage à un nouveau mode de scientificité : l'abandon d'un discours spéculatif d'inspiration philosophique au profit de méthodes d'investigation davantage tournées vers la quantification (Schweber, 2002) et le contact avec les enquêtés (par exemple, Stoetzel, [1946] 199I).

Cette nouvelle conception est à relier au contexte de l'après-guerre. Sollicitée par une demande étatique, la recherche se contractualise et prend en charge des problèmes liés à la reconstruction de la France (Pollak, 1976). La sociologie en tant que discours savant est concurrencée par des savoirs alternatifs dominants (existentialisme, marxisme, personnalisme...) qui lui dénient tout droit à dire quelque chose sur la société française (J. D. Reynaud, I96I). Le champ de la discipline est fractionné en plusieurs équipes de recherche groupées autour de «patrons » qui ont fait leurs débuts avant la guerre : Davy, Friedmann, Gurvitch, Stoetzel... qui luttent pour imposer leur conception de la scientificité, contrôler postes et ressources. Stoetzel promeut les sondages d'opinion; Gurvitch travaille à reconstruire «l'explication»; Friedmann développe une sociologie du travail dans les usines qu'il associe à un combat moral. Pour chacun, l’apport des Américains est une ressource qui légitime un changement. Jean Stoetzel incarne ce mode «d’affichage» (Blondiaux, 199I). Reste que cette référence à la sociologie américaine demeure profondément ambivalente. Les arguments généralement avancés soulignent la méfiance à l'égard du Plan Marshall et l’anti-américanisme des sociologues français, proches du PCF et du mouvement ouvrier. Mais ils n'épuisent pas l'explication de cette ambivalence.

Le présent article fait état de quelques résultats d'un récent mémoire d'HDR sur cette réception comprise comme une contribution à l'histoire de la sociologie en France (Marcel, 2010). Il s'appuie sur le corpus (environ 400 textes) des deux seules revues hexagonales traitant de sociologie dans les années 19401960: L’Année Sociologique et les Cahiers Internationaux de Sociologie. Cette investigation, 
«internaliste», observe la sociologie telle qu'elle se met en scène dans ces publications : un examen plus minutieux de cette réception des Américains par les Français, de leurs argumentaires, dévoile une sociologie française «reconstruite », sans forcément se refonder (Marcel, 2004a).

Selon nous, les textes étudiés restituent une cohérence globale et revêtent une dimension collective à travers les représentations qu'ont en commun les auteurs de ce que sont le social et sa «science». Quoique chaque auteur ait parmi les Américains ses propres «champions» et ses «repoussoirs», il est remarquable que tous manifestent une commune tendance à faire de la «société globale» la seule entité signifiante pour construire l'explication. C'est là une régularité frappante: le présupposé théorique consistant à considérer les faits comme le lieu privilégié de l'observation et de la compréhension d'une totalité sociale.

Si une telle posture a déjà été remarquée pour Gurvitch (Pollak, I988; Marcel, 200I), il n'est pas l'unique exemple, loin s'en faut, dès qu'il est question de commenter les Américains. Cet argument d'une société substantialisée comme totalité cohérente est le fil directeur de la «connaissance familière » à la lumière de laquelle ces auteurs sont lus, évalués voire utilisés (Pollak, 1988, p. 196). Nous posons l'hypothèse de l'existence persistante d'un paradigme - au sens de présupposés implicites, à partir desquels les chercheurs élaborent leurs théories - qui oriente pendant une décennie la compréhension des travaux produits outre-Atlantique. Ce présupposé tient probablement au fait que les Français déplorent dans le même temps l'éclatement de la recherche sociologique en France, et entreprennent de lire les Américains avec la conviction d’une nécessaire unité de leur discipline. Ceci les conduit parfois à percevoir de façon quelque peu abusive les travaux américains comme relevant d'une tradition intellectuelle nationale qu'on pourrait aisément identifier.

Ce qui suit expose des éléments extraits de l'analyse du corpus de tous les comptes rendus de L'Année, des articles, des études critiques et des comptes rendus des Cahiers. On présente ici les réceptions des contributeurs des pôles «opposés » : à savoir, celle du «groupe Gurvitch» incarnant la sociologie philosophique universitaire, et celle du «groupe Friedmann» représentant plutôt la sociologie empirique. 


\section{LA RÉCEPTION DE LA SOCIOLOGIE AMÉRICAINE DANS L'ANNÉE SOCIOLOGIQUE}

\section{I.I. LES AUTEURS RECENSÉS}

Tableau I : Auteurs américains les plus recensés et leurs «reviewers»

\begin{tabular}{|l|c|}
\hline $\begin{array}{c}\text { AUTEURS / RECENSEURS } \\
\text { ANNEEE DE RECENSION }\end{array}$ & TOTAL \\
\hline Linton R. & 5 \\
\hline Merton R. K. & 5 \\
\hline Whyte W. F. & 5 \\
\hline Jennings H. J. & 4 \\
\hline Kardiner A. & 4 \\
\hline Laswell H. D. & 4 \\
\hline Moreno J. L. & 4 \\
\hline Sorokin P. & 4 \\
\hline Lazarsfeld P. & 3 \\
\hline Warner W. L. & 3 \\
\hline
\end{tabular}

À noter la première place de Linton parmi les auteurs les plus commentés et l'absence de Parsons.

Merton occupe la première place ex æquo, au titre de spécialiste de la sociologie de la connaissance (voir Davy, 1952, p. 151). On a là vraisemblablement une conséquence de la publication de La sociologie au xxe siècle, dans lequel Merton offre un chapitre sur le sujet. La forte représentation de la sociométrie (Jennings, Moreno) traduit l'intérêt que l'étude des relations interpersonnelles suscita en France, investie par le groupe Gurvitch. Quant à Warner, c'est surtout son incursion dans ce qu'en France on nomme la «sociologie industrielle» qui retient l'attention. 


\section{I.2. LES CONTRIBUTEURS}

Tableau 2 et 3 : Contributeurs ayant recensé le plus d'auteurs américains et ayant le plus publié

\begin{tabular}{|c|c|c|c|}
\hline \multicolumn{2}{|l|}{ Tableau 2} & \multicolumn{2}{|l|}{ Tableau 3} \\
\hline CONTRIBUTEURS & $\begin{array}{l}\text { TOTAL AUTEURS } \\
\text { RECENSÉS }\end{array}$ & CONTRIBUTEURS & $\begin{array}{c}\text { TOTAL DES } \\
\text { RECENSIONS }\end{array}$ \\
\hline Davy.G & 26 & Davy.G & II \\
\hline Touraine A. & 24 & Touraine A. & 10 \\
\hline Friedmann G. & 13 & Friedmann G. & 9 \\
\hline Maisonneuve J. & 13 & Dufrenne M. & 6 \\
\hline Isambert F. & II & Naville P. & 5 \\
\hline Badinter R. & 10 & Lopstein J. & 4 \\
\hline Gurvitch G. & 10 & Szabo D. & 4 \\
\hline Naville P. & 9 & Sorre M. & 3 \\
\hline Dufrenne M. & 8 & Isambert V. & 3 \\
\hline Sorre M. & 8 & Margot-Duclot J. & 3 \\
\hline Lopstein J. & 7 & Bourricaud F. & 3 \\
\hline Poulat E. & 7 & Cazeneuve J. & 3 \\
\hline Szabo D. & 7 & Rolle P. & 3 \\
\hline Costa-Oeconomo J. & 6 & Tréanton J-R & 3 \\
\hline Isambert V. & 5 & Maisonneuve J. & 2 \\
\hline Margot-Duclot J. & 5 & Isambert F. & 2 \\
\hline
\end{tabular}

Le décompte consacre Georges Davy comme meilleur connaisseur de la sociologie américaine dans L’Année, selon les deux critères retenus. Ceci conduit à relativiser la dichotomie entre sociologie «spéculative» et sociologie empirique réputée plus axée sur les méthodes et travaux menés outre-atlantique. Les recensions de Davy, qui relèvent surtout de la sociologie générale et de la sociologie du droit, ont un but plus spéculatif que pratique. Il voit en les concepts de culture et de personnalité de base un moyen de concilier les apports de la psychologie et de la sociologie pour reconstruire à moindres frais l'explication. En matière de méthode, s'il accepte l'utilisation des questionnaires et interviews, et les salue comme représentatives d'une «new sociology» à la mode aux États-Unis, il rappelle que les systèmes de relations crées par l’action interindividuelle ne se nouent quau sein des structures qui les enserrent (Davy, 1956, I69-170). Ainsi s'explique la présence de Linton et Kardiner parmi les auteurs les plus commentés (voir supra). 
Outre Touraine (voir infra), les noms de J. Maisonnneuve, J. MargotDuclot $^{1}$ et dans une moindre mesure de F. Bourricaud (et de P. Kahn²) rappellent à quel point la présence des psychosociologues fut importante dans l'introduction et l'application de méthodes empiriques issues de la psychologie en sociologie. Ils furent des «passeurs» efficaces, comme le montrent aussi les travaux de recherche de P-H Maucorps et de P. Naville, tous deux issus de la psychotechnique (Martin et Vannier, 2002) au Centre d'Études Sociologiques. La pluridisciplinarité paraissait une nécessité pour reconstruire une explication solide des phénomènes contemporains.

\section{I.3. UNE RÉCEPTION PARTICULIÈRE}

Des Américains vus par les sociologues du travail

À titre d'illustration, nous avons choisi de présenter la réflexion que livrent les sociologues dits du «travail» dans L’Année. Le palmarès des contributeurs (voir supra) consacre Friedmann, V. et F. Isambert, P. Naville, A. Touraine, M. Crozier, J-D Reynaud et J-R Tréanton... parmi les «passeurs» de la sociologie américaine. Il faut y voir une convergence d'intérêts et d'objets de recherche (Chapoulie, 199I). Ce qui suit complète ce que nous avons déjà publié sur la question (Marcel, 2004a).

En nourrissant la rubrique «sociologie du travail», ils s'imposent comme les plus gros pourvoyeurs de comptes rendus, et font le plus gros effort collectif de réception de la sociologie américaine. Friedmann insiste sur l’apport de ce qu’il nomme la «sociologie industrielle» américaine, qui dans le domaine du façonnement psychologique et moral de l'individu apporte de nécessaires enquêtes et observations où les machines ont pénétré la vie des hommes (Chapoulie, 1991, p. 343). Mais ces études ne valent à ses yeux qu'à condition qu’elles permettent une compréhension simultanée de l'action du progrès technique sur la mentalité humaine considérée dans son individualité certes, mais

I J. Margot-Duclot était aussi un psychologue proche de Jean Stoetzel et Roger Daval (renseignement fourni par B. Brévan).

2 Proche manifestement de Gurvitch il signe beaucoup de notices dans les Cahiers Internationaux, dont un nombre non négligeable fait l'exégèse des travaux de ce dernier, et fait partie du CES où il organise des séminaires de sociologie de la connaissance (Marcel, 2005). 
pourvu que celle-ci soit replacée dans les groupes où elle est plongée, tâche qu'il se propose de mener (Marcel, 2004a, p. 59) 3

Dans le prolongement de l'idée qu'ils se font de l'insertion des hommes dans leur milieu, Friedmann et son équipe travaillent donc à l'élaboration d'une psycho-sociologie du travail à la chaîne, où celle-ci doit être considérée comme un fait à la fois historique, technique, physiologique, psychologique et social. Ce programme est du reste présenté dans le tome 2 de la livraison de 1940-1948 de L’Année (Friedmann, 1949, p. 713).

Le compte rendu que Touraine donne du livre de Friedmann Où va le travail humain ${ }^{4}$, prend le relais de cette déclaration de principe, précisant que les usines américaines sont un mélange d’audace technique et de timidité intellectuelle face à un environnement technique qui ne «relâche pas sa pression » (Touraine, 1952a, p. 420). C'est une des tâches de la sociologie industrielle que de «préciser» comment «les structures globales» «pénètrent» et «modifient» des questions telles l'adaptation au rythme de travail, l'intégration aux équipes d’atelier, la fluidité de la main d’œuvre, les rapports avec les hiérarchies formelles etc. (ibid., p. 42I). Les Américains ont le tort de se cantonner à l'étude de l'entreprise considérée comme un milieu fermé, isolé du reste de la société.

On peut dire quavec les premières recensions du volume 1940-1948 les principaux axes de réflexion qui vont guider cette réception sont posés. Elle s'accompagne d'un positionnement qui se définit souvent «contre » ce qu'il ne faut pas faire, dévoilant les catégories de perception des recenseurs, calquées sur la façon dont ils identifient les différents courants américains.

\section{I.3.I. L'influence du milieu technique sur les ouvriers}

Friedmann associe cet objet d'étude à «l'école américaine des anthropologues sociaux » incarnée par R. et L. Lynd ; W. L. Warner (Friedmann, 1949, p. 762) dont la méthode appliquée aux collectivités industrielles a fait ses preuves. Il compare ces travaux à ceux de «l'école des Industrial Relations », plus axée sur l'aspect psycho-social des relations de travail. Si bien qu'il s'autorise à parler de

3 Pour une histoire de ce groupe, voir Chapoulie (1991); Tréanton (1991, 1992); Marcel (2005).

4 Paris, Gallimard, 1950. 
«sociologie industrielle» au singulier, qui caractériserait un «esprit» ou une tradition nationale typiquement américaine.

À noter que le commentaire de la méthode anthropologique d'observation des ateliers est sans doute à rapprocher de celui que font les sociologues du travail français des travaux des sociologues de Chicago, qui faisaient partie, avec Warner, du Committee on Human Relations in Industry de l’Université de Chicago (Chapoulie, I99I, 343).

- Du côté de l’anthropologie sociale :

La réception de W. F. Whyte ${ }^{5}$ par exemple, un auteur parmi les plus commentés sur la décennie est emblématique pour les Français du traitement par les Américains de la question de l'influence du milieu technique.

La première recension qui le concerne porte sur des travaux du Commitee on Human Relation in Industry et figure aux côtés d'une série d'autres publications de Hughes, Warner, Starr, A. Davis par exemple, qui interrogent les relations entre travailleurs et les conflits dans travail. Le compte rendu est typique de cette période, durant laquelle les sociologues de Chicago sont lus par le «groupe Friedmann » avec une bienveillance condescendante.

Si l'intérêt du travail de Whyte «réside en partie dans les nombreuses descriptions concrètes qui y sont incluses» (Lopstein, I949, p. 800), et si les causes des conflits suite à la concentration du travail (Warner et Low), l'introduction de travailleurs noirs (Hughes) et la description de la mentalité ouvrière (Davis) sont bien rendues, la conception de la lutte des classes reste «bien étroite» (Starr). Lopstein ajoute qu'en dépit d'une foi naïve des auteurs en la possibilité d'une harmonie entre travailleurs et décideurs obtenue grâce aux progrès des techniques d'organisation, la psychologie de l'homme au travail des Américains fournit des «matériaux d’un puissant intérêt» (ibid., p. 802) qui ont le mérite d'attirer l'attention sur les maux de l'industrie (ibid, p. 800). Mais si ces études de cas sont intéressantes car elles permettent de détailler les problèmes dans les relations de travail lors des changements techniques et d’organisation du travail, elles manquent toutefois l'essentiel car «les aptitudes

5 Quoique la postérité l'ait ensuite consacré et identifié comme l'auteur de Street Corner Society, c'est en effet d'abord pour son travail en sociologie industrielle qu'il est recensé dans L'Année. 
d'un ouvrier $[\ldots]$ sont fonction de leur capacité de se réaliser socialement dans un groupe $e^{6} \gg$ (Touraine, 1952a , p. 422). À ce titre, le volume de Whyte surenchérit Touraine, est prometteur mais insuffisant:

La psycho-sociologie industrielle ne se développera qu’en rompant avec les prémisses utilitaires de ses premières recherches [...] trop souvent détournée «de la science explicative vers les considérations normatives», et la «limitent» encore, dans beaucoup d'universités américaines, à définir «une sorte d’art des relations industrielles», qui n'est pas sans ambiguïté (p. 421-422).

Une deuxième réception typique de ce courant anthropologique est celle des travaux de Warner. Elle est par exemple le fait de G. Friedmann qui compare The Social System of the Modern Factory à des textes de Mayo entre autres. Le travail des Américains est présenté comme permettant de combler la carence d'une sociologie marxiste qui «n’a pas systématiquement prospecté un domaine» : celui des groupes humains que les hommes forment au travail et qui sont soumis à l'évolution des techniques de production et de la division du travail (Friedmann, I949, p. 76I). Aussi, bien que les travaux de Warner puissent être «un appoint d'information objective», ses observations effectuées sur les ouvriers au travail ne peuvent trouver sens que rapportées à l'ensemble de l'environnement social (p. 765).

- L'étude psycho-sociologique des relations de travail:

Sous ce vocable, il faut sans doute placer la réception du travail du Mouvement des Relations Humaines, et de toutes les enquêtes qui s'y rattachent et s'en réclament. On peut même dire que la perception de la sociologie industrielle américaine dans L’Année est une surtout une prise de position face au travail de Mayo et de son équipe.

Très bon connaisseur des travaux de Mayo, qu'il a même rencontré en 1939 (Desmarez, 2004, p. 105) Friedmann le commente abondamment (I946, 1950a, 1950b par ex.). Il estime que Mayo effectue un pas décisif vers la reconnaissance de l'importance des facteurs sociaux dans l'entreprise, la critique de la rationalisation techniciste et l'humanisation des chaînes (Desmarez, 2004, p. I05). Mais il le critique aussi durement: en soulignant l'importance des facteurs 
psychologiques et sociaux sur le rendement, Mayo et ses collaborateurs ont donné naissance à une «managerial sociology» utile surtout à des services de direction et de personnel (Friedmann, 1950b, p.47). Toutes les techniques américaines d'humanisation du travail, - telles que le «counselling», qui consiste à recevoir individuellement les ouvriers pour leur donner la possibilité de s'exprimer - ne peuvent empêcher une dégradation de l'énergie et de la volonté, une atrophie des personnalités, liées à l'extension de la fabrication de série.

Ainsi, Industrial Psychology and its social foundations de Milton Blum, du département de psychologie de la «School of Business and Civic Administration» de New York, est un livre que Friedmann tient en estime, mais il considère que la conception de l'entreprise qui émane des enquêtes de Hawthorne est «isolée et suspendue dans une sorte de vacuum social» (Friedmann, 1952, p. 436). À l'actif, il souligne que Blum relie la question de la satisfaction au travail aux attitudes que l'ouvrier adopte à son égard, mais aussi à d’autres facteurs qui ont trait à la vie en général.

La réception des autres sociologues du travail sur cette catégorie de travaux s'inscrit dans la lignée de Friedmann: se focaliser sur les problèmes d’ordre psychologique que pose le travail à la chaîne aboutit à réduire les cadres sociaux à des traits psychologiques personnels ou, au mieux, inter-personnels, qui ne disent rien des structures plus générales dans lesquelles s’inscrit l’activité de travail.

Touraine commente un livre d'H. Ronkien et de P. Lawrence, se réclamant de l'enseignement de Mayo, qui s'efforce de suivre les mécanismes de l'introduction du changement dans le système technique et social de l'entreprise, du point de vue des communications et des relations humaines : plus précisément les conflits d'attribution et les défauts de communication entre les personnes responsables lors de l'introduction d'une nouvelle fabrication. L'étude reste limitée à un petit nombre de groupes et est plus utile aux utilisateurs de la sociologie industrielle quaux sociologues. Touraine ajoute quau surplus elle n’apporte pas grand-chose aux connaissances sur cette question (Touraine, 1956) en sorte que si la richesse de la pensée de Mayo est attestée par le nombre d'études qu’elle a inspirées, elle n’en garantit nullement le succès. 
Par extension, ce sont toutes les études de psychologie industrielle qui sont passées au crible de cette critique, qu’elles relèvent de ce que les Français appellent des études de «Small Groups», ou des enquêtes par sondage inspirées de la sociologie de Columbia. J.-D. Reynaud commente par exemple un volume de psychologie industrielle publié par des psychologues de l’Université de Pittsburgh et affirme que les problèmes sont, trop souvent, choisis en fonction de leur aptitude à être traités par ces techniques déjà acquises. Le problème que pose les relations industrielles n'étant pas celui des moyens techniques, mais celui des types de relations qui peuvent s'établir (Reynaud, 1952), il convient donc d'assouplir les instruments (par ex. les interviews) et de quitter le laboratoire pour aller vers le monde réel. De même, Reynaud reproche-t-il à E. Chambers de tirer des analyses qui relèvent surtout de la psychologie individuelle et de simplifications psychologiques (Reynaud, 1953, p. 455), des conclusions sociales qui mériteraient discussion. Il faudrait une «analyse des cadres sociaux de la production qui fait ici totalement défaut» (p. 456).

\section{I.3.2. Les relations de travail (liées à la division technique des tâches, hiérarchiques, etc.)}

Les recensions débouchent sur la critique d'auteurs trop soucieux de promouvoir la «paix industrielle», sous-estimant la place des conflits et la signification de leurs enjeux ramenés à la globalité de la société. Par extension cette grille de lecture commande la perception de la littérature relative au syndicat et aux mouvements ouvriers. Derrière cette question du rôle du syndicat et des conflits, c'est la conception américaine des classes sociales et de leurs rapports qui est critiquée.

- Syndicats et conflits : un aperçu de quelques recensions :

«Concepts vagues ou mal appropriés» (Tréanton, 1953, p. 444), livre qui n’apporte rien parce qu'il se concentre sur les vieux souvenirs et les anecdotes pour mieux les apaiser, en dépréciant les plus révolutionnaires (Crozier, I953), ignorance du point de vue des victimes de la productivité et de leur mécontentement suite à une fermeture d'usine (Tréanton, 1955)..., la conception améri- 
caine a pour unique mérite de fournir un matériau éclairant, utilisable pour autant qu'on ne prenne pas en compte les concepts utilisés.

Une place particulière doit être faite à Michel Crozier dont le syndicalisme américain est la spécialité depuis son séjour aux États-Unis de 1947, d’où il a ramené son livre: Syndicats et ouvriers d'Amérique . L'analyse de la réception de Crozier montre qu'il adopte un ton critique sur l'ignorance des aspects conflictuels de la vie syndicale. Bien qu'idéologiquement plus américanophile, il publie des comptes rendus en phase avec les autres sociologues du travail. À propos de Heritage of Conflict, labor relations in the Nonferrous Metals Industry up to I930, de Jensen, il rappelle que l'intérêt d'une histoire du mouvement ouvrier aux ÉtatsUnis serait l'analyse d'un mouvement ouvrier extrêmement précoce et violent qui se dégrade en syndicalisme d’affaires (Crozier, 1953, p. 47I). Or Jensen se borne à faire le récit des anecdotes et des vieux souvenirs et déprécie plus les révolutionnaires qu’il n’explique leur échec.

- Conflits et «paix industrielle»: W. F. Whyte dans le colimateur:

La notice sur le travail de W. F. Whyte, Pattern for Industrial Peace, est emblématique aussi de ce type de réception. V. Isambert signale que, quoique Whyte présente son enquête comme un «case study», c'est en réalité «un essai de portée générale sur les relations industrielles, précédé de l'étude d’un cas qui éclaire chacune des assertions théoriques», mais qui manque son but (V. Isambert, I953, p. 476). Elle loue l'auteur de faire un récit plein de vie, de sympathie pour ceux qu'il observe, de donner des détails concrets et parlants, néanmoins:

La relation entre une situation sociale d'entreprise et la situation sociale globale est totalement ignorée. Enfin est-il besoin d'ajouter que, pour habituelles qu'elles soient chez les sociologues américains, les conclusions pratiques d'un ouvrage sociologique, avec ses conseils aux patrons et aux leaders syndicaux, ne laissent pas d'étonner le lecteur français? (ibid., p. 478). 


\section{- La critique de l'analyse des classes sociales aux États-Unis :}

De cette absence de prise en compte des enjeux globaux des conflits découle logiquement la critique de l'étude des couches sociales. Le meilleur exemple de réception sur la question est sans doute la synthèse de Touraine ${ }^{8}$. Il y signale l'apparition de nombreuses études récentes sur les classes sociales, mais déplore que toutes ou presque «renoncent d'emblée à préciser l'unité des fondements objectifs de la classe et de la conscience de classe affirmée par Marx » (Touraine, 1952b, p. 219). Touraine identifie deux écoles : d'un côté les objectivistes comme Pareto, Veblen ou les «anthropologues sociaux» à la Dollard «Warner et son équipe des Yankee City Series» qui «définissent l'appartenance à une classe par la situation hiérarchique, le statut, le rôle social de l'individu» (ibid., p. 219); de l'autre les «subjectivistes» qui comme Sombart choisissent comme critère d'appartenance à une classe certaines attitudes et en particulier l'auto-affiliation. D’autres, comme Sorokin, ont essayé de dépasser cette dichotomie et n’y sont pas arrivés. Tout cela vient d'une confusion entre les notions de «strate» ou groupe social, et de «classe sociale». La classe «doit se définir par des attitudes à l'égard des problèmes économiques, politiques et sociaux et, avant tout, par l'affiliation consciente des individus à une classe qu'ils désignent eux-mêmes » (ibid., p. 220).

Par ailleurs Touraine pense que l'étude des strates est renvoyée à l'anthropologie sociale et à l'économie, alors qu'il appartient aux psychologues d'analyser les classes sociales. C'est la position de Centers sur la question qui lui semble partagée par beaucoup de chercheurs américains, notamment Cantril et Kornhauser9. Dans ce cas, ce sont les attitudes sociales de groupes constitués qui sont analysées et l'expérience conduit à choisir les groupements professionnels comme base d'étude. L'union des orientations politico-économiques est affirmée par la théorie des groupes d'intérêt, qui suppose que le statut et le rôle d’une personne par rapport au processus économique d’une société lui

8 Nous avons déjà donné quelques éléments de cette réception dans Marcel, 2004b.

9 II cite: H. Cantril, «Identification with social and economic class», Journal of abnormal psychology (1943, p. 74-80); A. W. Kornhauser, «Analysis of class structure in contemporary American society. Psychological bases of class divisions», in Industrial conflict: a psychological interpretation, sous la direction de G. W. Hatmann et T. Newcomb, New York (1939); R. B. Cattell, «The concept of social status», Journal of abnormal and social psychology (1948, p. 293-308). 
imposent certaines attitudes, valeurs et intérêts relativement à son rôle et à son statut dans le domaine économique mais aussi politique.

Le corps du livre de Centers exposerait les corrélations étroites qui unissent l'affiliation à une classe sociale et les orientations politiques-économiques, ainsi que la situation professionnelle. Il livrerait des analyses «intéressantes » voire «pénétrantes » qui apportent des précisions sur la base sociale d’attitudes comme les préjugés raciaux, l'appartenance religieuse, la conception du rôle social de la femme, ce qui conduirait à reconnaître l'existence de classes aux États-Unis, dont beaucoup nient l'existence.

Mais l'étude appelle des réserves: les classes distinguées ne sont que des abstractions, réduites à des niveaux dans la hiérarchie sociale. L'étude porte sur l'Amérique à un moment donné, en faisant abstraction de sa situation historique précise. Il faudrait des données sociales objectives, pour permettre à la sociologie des classes sociales de «déterminer les rapports des forces de production, des rapports sociaux de production et de la conscience de classe liés dans l'observation historique » (ibid., p. 222). Impossible de séparer les structures concrètes de la société et les attitudes sociales, sans quoi on ne voit dans les attitudes sociales que les transcriptions sur le plan de la conscience individuelle des structures objectives.: «Le problème est, en fait, ailleurs, dans l'analyse de ces structures elles-mêmes, des rapports sociaux de production $[. .$.$] que$ l'école psychologique américaine refuse de considérer » (idem).

Du côté de l'étude objectiviste des classes sociales, on part souvent du concept de statut socio-professionnel. Les résultats des sondages proposent des échelles répartissant hiérarchiquement certaines professions. Hall et Jones ont ainsi fait placer I38 professions en 9 classes définies en fonction de la catégorie de personnes que sont amenés à fréquenter ceux qui l'exercent. Le statut socio-économique est pour Touraine une notion riche très corrélée aux attitudes collectives. Il commente ainsi les travaux d’Eysenck, Geiss et Benney qui dégagent les rapports entre statut et opinions politiques: 
L'intérêt de toutes ces études positives est indiscutable, et l'on doit regretter que des sondages et des mesures statistiques ne soient pas consacrés à ces problèmes en France. Est-ce à dire pourtant, que l'on souhaite voir entreprendre ici des études sur le modèle des travaux anglais et américains? Assurément non, surtout si l'on s'inquiète de l'apport de telles études au problème des classes sociales $[\ldots]$ Le nombre et le succès des corrélations établies ne doivent pas tromper. Jamais n'est établi un rapport entre deux réalités distinctes, dont l’une serait la classe sociale, définie précisément (ibid., p. 224).

«Ainsi, le raffinement des techniques employées n’empêche pas la faiblesse des conclusions et l'inutilité quasi totale de ces recherches pour l'étude des classes sociales » (ibid., p. 225). Plutôt que de se contenter d'une échelle d'estime faite avec description chiffrée, il faut considérer le système des classes comme «un certain état des rapports sociaux de production» (idem). Pour chercher comment les formes de l'appropriation sont en relation avec les attitudes et les opinions ou les expliquent, Touraine demande qu'on ajoute au modèle un indice d'estime et un indice de prestige. En somme, le travail des Américains mobilise un appareil empirique d’investigation élaboré sans faire réellement progresser l'explication car il passe à côté de l'essentiel.

Ce leitmotiv de la nécessité d'une explication passant par la totalité de la société apparaît donc comme un cadre d'analyse destiné à replacer pour les interpréter les relations de travail entre individus au sein de l'entreprise. La réception qui suit, tirée des Cahiers Internationaux de Sociologie, s'efforce aussi, quoique différemment, de tirer de la lecture des auteurs américains les éléments susceptibles de comprendre et penser des phénomènes «microsociologiques » dans le cadre d’analyse de la société dans son entier. 


\section{LA RÉCEPTION DE LA SOCIOLOGIE AMÉRICAINE DANS LES CAHIERS INTERNATIONAUX DE SOCIOLOGIE}

\section{I. PALMARÈS DES AUTEURS RECENSÉS}

Tableau 4: Auteurs américains les plus cités durant la décennie

\begin{tabular}{|l|c|}
\hline \multicolumn{1}{|c|}{ AUteUR } & $\begin{array}{c}\text { NOMBRE DE } \\
\text { CITATIONS }\end{array}$ \\
\hline Moreno J. L. & 24 \\
\hline Linton R. & 20 \\
\hline Malinowski B. & 19 \\
\hline Kardiner & 17 \\
\hline Parsons T. & 17 \\
\hline Sorokin P. & 17 \\
\hline Znaniecki F. & 15 \\
\hline Merton R. K. & 14 \\
\hline Lewin K. & 12 \\
\hline Mead M. & 11 \\
\hline Benedict R. & 10 \\
\hline Kluckhohn C. & 10 \\
\hline Mead G. H. & 10 \\
\hline Lazarsfeld P. & 9 \\
\hline Sumner W. G. & 9 \\
\hline Warner W. L. & 9 \\
\hline
\end{tabular}

Ce tableau comptabilise le nombre de fois où un auteur américain est cité au moins une fois dans un texte du corpus.

Nous avons retenu les 16 auteurs américains les plus commentés et cités. Sont comptabilisées les références données dans les textes écrits par des auteurs américains : leur faible nombre ne change pas beaucoup les places relatives. Les différences majeures avec le palmarès établi dans L’Année sont les suivantes : alors que les travaux de Jennings, Laswell et Whyte y sont souvent recensés, ces auteurs ne sont pas très présents dans les Cahiers. G. et M. Mead, Parsons, Sumner, Lewin, Malinowski, et Znaniecki, souvent évoqués dans les Cahiers, n’ont pas la vedette dans L’Année.

À ces différences près, ce sont les mêmes qui sont consacrés par les lectures des contributeurs: Linton ( $\mathrm{I}^{\mathrm{er}}$ dans L’Année et le $2^{\mathrm{e}}$ plus cité ici!); Moreno ( $2^{\mathrm{e}}$ dans l'Année ex æquo et $\mathrm{I}^{\mathrm{er}}$ dans les Cahiers) ; Kardiner (respectivement $2^{\mathrm{e}}$ ex æquo et $4^{\mathrm{e}}$ ); Sorokin $\left(2^{\mathrm{e}}\right.$ et $\left.3^{\mathrm{e}}\right)$; Merton ( $\mathrm{I}^{\mathrm{er}}$ ex æequo et $\left.8^{\mathrm{e}}\right)$. Ainsi, la réception de la sociologie américaine dans les revues françaises a surtout concerné l'anthropologie cultu- 
relle et la sociométrie, comme l'illustre le «duo gagnant» Linton/Moreno, avec mention spéciale à Moreno dont la sociométrie paraissait à Gurvitch être une pierre dans la reconstruction de la sociologie telle qu'il l'envisageait. La présence d'un auteur comme Warner dans les deux corpus s'explique par le choix de son sujet d'étude: les classes sociales - qui suscitent une attention soutenue. La forte présence de Malinowski (assez peu «américain» il est vrai) est un peu ironique puisque la plupart du temps, il est donné comme l'exemple d’un fonctionnalisme naïf et réducteur qu'il importe de ne surtout pas imiter... Cette comparaison dont émerge ces similitudes suggèrent qu'il y eut quelques grands «courants» de réception, dont le culturalisme, surtout, constitue un moment-clef.

\subsection{LE PALMARÈS DES CONTRIBUTEURS}

Tableau 5 : Les contributeurs et leurs publications dans les Cahiers ${ }^{10}$

\begin{tabular}{|l|c|l|c|}
\hline \multicolumn{1}{|c|}{ NOM DU CONTRIBUTEUR } & TOTAL & \multicolumn{1}{|c|}{ NOM DU CONTRIBUTEUR } & TOTAL \\
\hline Gurvitch G. & 8 & Chaix-Ruy & I \\
\hline Balandier G. & 6 & Chombart de Lauwe P-H & I \\
\hline Kahn P. & 6 & Clignet R. & I \\
\hline Bastide R. & 4 & Cuvillier A. & I \\
\hline Bourricaud F. & 4 & Dumazedier J. & I \\
\hline Touraine A. & 4 & Duveau G. & I \\
\hline Dufrenne M. & 3 & Haudricourt et Granai & I \\
\hline Isambert F. A. & 3 & Jennings H. H. & I \\
\hline Maisonneuve J. & 3 & Lefort C. & I \\
\hline Mercier P. & 3 & Lynd R. & I \\
\hline Moreno J. L. & 3 & Lyotard J-F & I \\
\hline Sorokin P. & 3 & Maitre J. & I \\
\hline Badinter R. & 2 & Margot-Duclot J. & I \\
\hline Cazeneuve J. & 2 & Maucorps P-H & I \\
\hline Crozier M. & 2 & Mortel R. & I \\
\hline Friedmann G. & 2 & Paulme & I \\
\hline Girod R. & 2 & Pauvert J-C & I \\
\hline Janne H. & 2 & Rodgers M. & I \\
\hline Mitrani N. & 2 & Rose A. & I \\
\hline Vexliard A. & 2 & Spenlé & I \\
\hline Angell R. & $\mathrm{I}$ & Williams R. & I \\
\hline Aron R. & $\mathrm{I}$ & Wirth L. \\
\hline Avigdor & $\mathrm{I}$ & Zazzo R. \\
\hline Burgess E. & $\mathrm{I}$ & Znaniecki F. \\
\hline Cahn P. & $\mathrm{I}$ & & \\
\hline
\end{tabular}

10 On fait ici pour chacun la somme des articles, études critiques et comptes rendus. 
Dans les Cahiers, on trouve différents types de textes, à la différence de L’Année où sont principalement publiées des notices, mémoires originaux mis à part. Outre celles de Moreno et Sorokin (avec qui Gurvitch a été lié aussi un temps), il apparaît difficile de caractériser la participation des quelques Américains ayant écrit dans les Cahiers. La plupart de leurs articles ne sont pas des textes originaux. Il n'est guère aisé de trouver une réelle logique intellectuelle à la présence d'un tel plutôt qu'un autre, si ce n'est sans doute quelque lien personnel à un moment donné. Il est néanmoins remarquable qu’ils aient eu une tribune où transmettre certaines de leurs idées à un lectorat français.

En ce qui concerne les autres contributeurs, sans surprise, c'est Gurvitch qui est consacré comme le plus actif. Nous avons ailleurs tenté de caractériser la réception que Gurvitch fait des travaux des Américains (Marcel, 200 I Marcel, 2004a). Fondateur et directeur de la revue, il en fait un tremplin pour promouvoir sa conception du social. L'existence des Cahiers, ainsi que sa réception des Américains et ses choix éditoriaux peuvent être reliés à son projet général de reconstruction de ce qu'il appelle «l'explication en sociologie». Nombre d'articles portent sa marque, que les auteurs se réfèrent directement à lui ou non.

De ce fait, son projet constitue un cadre de compréhension sans lequel tout ce qui suit n'est pas intelligible: Gurvitch défend une conception de la sociologie dans laquelle la société est pensée comme en perpétuel changement, prise dans un mouvement de déstructuration et de restructuration permanent (l'article «Hyper-empirisme dialectique», dans le volume i5 de 1953). Cette dynamique dévoile l'existence de «phénomènes sociaux totaux» (par exemple la stratification sociale), lesquels traversent de part en part différents secteurs dans lesquels s'expriment le social et qui vont des ensembles les plus vastes aux tréfonds des consciences individuelles, en passant par les relations entre groupes et individus. Ainsi s'explique le fort investissement théorique sur le concept de «classe sociale», objet d'un certain nombre d'articles du corpus : «Qu’est-ce qu’une classe sociale? » écrit par Sorokin dans le volume 2, «Groupement social et classe sociale» donné par Gurvitch dans le volume 7, ou «Classe sociale et statut socio-économique » de Touraine dans le volume i I. 
C'est là, comme on sait, une arme de combat contre des sociologies concurrentes (Friedmann, Stoetzel, Aron) mais aussi des discours tels que le marxisme, la psychanalyse, le structuralisme et la phénoménologie. Dans cette configuration, l'enjeu est d'abord de théoriser l'immanence entre l'individuel et le collectif, ce qui se traduit par la mise au point des fameux «paliers» qui sont des points d'observation (la surface morphologique ou écologique par exemple). Mais cette observation doit permettre au sociologue de constater à tous les niveaux de la réalité sociale - tous les paliers - la «réciprocité des perspectives» qui pose que consciences individuelles et consciences de groupes sont immanentes. Ainsi s'expliquent la polémique que Gurvitch lance contre Lévi-Strauss pour critiquer férocement le concept de «structure sociale » qui est concurrent de celui de «palier» de ce point de vue, et la profonde aversion qu'il manifeste aussi pour la théorie de l'action de Parsons qui prend en charge cette idée d’immanence (Marcel, 2004b). Manque toutefois à Gurvitch - qui à la différence de Lévi-Strauss et Parsons ná pas d'outils efficaces pour l'abstraction analytique - afin de compléter son édifice ce qu’il nomme une «microsociologie» susceptible d'étudier les phénomènes sociaux au niveau «intermental» des relations entre individus dans les petits groupes («Microsociologie et sociométrie» dans le volume 3 de 1947). C’est dans cette optique qu'il faut comprendre, le «mariage» de Gurvitch et Moreno, et la présence de celui-ci, ainsi que celle de Jennings, parmi les contributeurs, car ils apportent, avec la méthode du psychodrame, des protocoles de recherches efficaces que Gurvitch entend intégrer dans son cadre conceptuel.

La $2^{\mathrm{e}}$ place de Balandier parmi les contributeurs s'explique bien sûr par ses liens avec Gurvitch, mais sa place est originale dans cette réception car il essaie d'expliquer les phénomènes de domination dans les pays colonisés à l'épreuve des travaux des anthropologues américains sur les minorités ethniques opprimées, et en particulier au moyen du concept d'acculturation. À ces réflexions s'ajoutent des questions théoriques et épistémologiques qui sont régulièrement débattues dans les textes des Cahiers (voir l'article dans le volume 2i sur la question de l'explication en ethnologie). 
La place de choix de Paul Kahn s'explique de même par ses liens avec Gurvitch. Il signe beaucoup de textes dans les Cahiers Internationaux, dont un nombre non négligeable fait l'exégèse des travaux de ce dernier. Remarquable est aussi la présence, parmi les plus importants contributeurs, outre Balandier et Gurvitch, de personnes comme Bastide, Dufrenne, Cazeneuve. La réception de ces auteurs est avant tout théorique. Ce sont tous des philosophes de formation, devenus spécialistes dans une discipline étudiant le social. Ils sont liés d'une manière ou d'une autre à la sociologie universitaire de la Sorbonne. Leurs contributions relèvent pour partie de textes qui essaient, à partir d'un examen critique des théories américaines, de chercher des éléments susceptibles d'aider à poser les fondements d'une sociologie plus psychologique. Ils ont aussi pour sujets des réflexions plus générales, sur la causalité en sociologie (Bastide), ou la connaissance (Cazeneuve).

Un autre groupe de contributeurs se situe en quelque sorte à la frontière entre les questionnements de cette science sociale universitaire et la recherche sociologique en train de se faire, notamment au Centre d'Études Sociologiques. Leur bagage théorique les autorise à faire des incursions dans la réflexion épistémologique, alors qu'ils mènent en même temps des enquêtes de «terrain ». Parmi eux, outre les sociologues du travail, écrivent des personnes comme Maisonneuve, Isambert, Bourricaud, qui réfléchissent à l'utilisation de concepts américains. Touraine se penche sur des notions telles que le rôle, le leadership, le groupe de référence... (Touraine, 195 I), Bourricaud s’interroge sur la portée du concept de «caractère national» (Bourricaud, 1952a), ou de «lien personnel» (1953b). Ils offrent aussi des contributions plus générales, par exemple sur les limites de l’analyse des groupements «intermédiaires» (Bourricaud ${ }^{11}$, 1952b et Touraine, 1954, principalement).

II Bourricaud se fait par ailleurs une spécialité du commentaire de Parsons, que nous n'avons pas la place de présenter ici. 
Enfin, un dernier groupe de textes révèle la présence d'un ethnologue (Mercier) et de psychosociologues (Maucorps, Avigdor, Spenlé, Mitrani). Ce sont des comptes rendus d'enquête qui montrent les applications sur études de cas de concepts liés aux phénomènes d'acculturation, à l'utilisation du «rôle», des «stéréotypes», ou des méthodes de la sociométrie. Ces réceptions opèrent un remodelage des frontières disciplinaires autour de réflexions inspirés de la science sociale américaine.

Ce corpus des Cahiers est une tentative pour repenser les fondements d'une sociologie susceptible de prendre en charge le vécu des individus tout en préservant les avantages d'une scientificité qui passe par la prise en compte de la globalité du cadre social dans laquelle les phénomènes sociaux sont observés. À cette entreprise, dont Gurvitch se veut le chef d'orchestre, tous participent à leur façon. Les stratégies intellectuelles et/ou institutionnelles de Gurvitch, que les études d'histoire de la sociologie française ont l'habitude de mettre en avant pour expliquer l'influence qu'il a pu avoir, ne nous semblent pas des explications suffisantes pour expliquer le caractère apparemment «allant de soi » de ce discours : ne jamais négliger le cadre social global dès qu’on tente d'expliquer un fait. Il en découle que nombre d'articles des Cahiers sont des tentatives de reformulation conceptuelle qui vont dans ce sens. Certains d'entre eux peuvent être perçus comme une mise à l'épreuve dont l'ethnologie et la psychologie sociale donnent l'exemple en abordant des problèmes concrets.

À titre d'exemples, nous livrons deux de ces tentatives de reformulations : rendre la sociologie plus «psychologique», et rediscuter les frontières entre les disciplines.

\section{3. «PSYCHOLOGISER»LA SOCIOLOGIE \\ Discussion théorique autour de l'anthropologie culturelle}

Cette ambition renvoie à des articles qui examinent les liens possibles entre la sociologie et la psychanalyse (Bastide dans le $2^{\mathrm{e}}$ volume), l'existentialisme (Dufrenne dans le rer volume), ou encore la phénoménologie (volume ı). Ces textes sont surtout écrits par des philosophes, et vont chercher dans les travaux américains une inspiration pour intégrer le social vécu par l’individu dans l'explication sociologique des phénomènes humains généraux. La production américaine est alors une sorte de boîte à outils épistémologique dont il faut 
améliorer les performances pour mieux définir les frontières entre disciplines. Deux auteurs émergent dans le corpus : Kardiner et Mead.

Les discussions autour des apports de «l'anthropologie culturelle menées par Dufrenne (Dufrenne, 1952), ou encore les exégèses faites par Lefort des travaux de Kardiner par exemple (Lefort, 1951), peuvent être comprises ainsi. Les travaux de ces Américains étaient sans doute une ressource assez souple pour offrir des pistes de réflexion pour cette entreprise de «psychologisation » de la sociologie. Les théories fonctionnalistes de Parsons et de Malinowski, deux auteurs souvent pris comme exemples repoussoirs, étaient non seulement idéologiquement suspects (perçues souvent comme «réactionnaires») mais aussi théoriquement moins acceptables. Parce que d’abord concurrentes - elles donnent une solution à la grande question épistémologique qui taraude les Français (penser l'individuel en rapportant les significations qui s'y rattachent à l'ensemble de la société) -, mais aussi trop bien «bouclées» pour pouvoir autoriser emprunts et interprétations relativement libres. Le concept de culture et ses avatars étaient suffisamment flous et malléables pour traiter divers objets, sans donner l'impression de «s'inféoder » théoriquement et idéologiquement. Cette réception rejoint celle de Davy dans L’Année, soucieux d’isoler et de justifier «la part de l'individuel» dans le discours sociologique. Ce sont les fondements théoriques de cette psychologisation et ses conditions de possibilité qui sont recherchés au travers de la lecture critique des Américains.

\subsection{Discussion de l'apport de Kardiner}

Dufrenne explicite ce qui fait l'intérêt du travail de Kardiner : traiter psychologiquement des faits culturels, ce qui ne requiert pas d'introduire dans l'étude du social l'individu comme tel «mais l'humain, afin de comprendre le social non seulement comme ce qui se réalise à travers des hommes, mais comme ce qui est vécu et agi par eux» (Dufrenne, 1952, p. 27). La culture étant l'aspect humain du social, la position intellectuelle défendue par Kardiner permettrait de dépasser une aporie de la sociologie: déshumaniser le social pour le saisir dans sa spécificité ou réintroduire l'humain dans le social pour le saisir comme vécu. 
L'examen de la culture invite donc la sociologie à se psychologiser. [...] Car il y a une réalité propre de la culture, qui s'impose aux comportements individuels, et une logique propre de cette culture qui se déploie en dehors des individus, bien que la culture nait de sens que par leurs comportements [...] Mais en retour la culture exprime aussi la réaction de l'humain aux exigences du social (ibid., p. 30).

Dufrenne se concentre sur les institutions qui s'imposent aux hommes, mais reflètent aussi ce qu'ils pensent et font. Il s'efforce de voir comment la culture agit sur l'individu pour le former comme dans les travaux sur l'éducation de M. Mead, et dans le Paysan Polonais, et comment l'individu peut réagir à la culture pour la modifier. C'est ce double rapport aux institutions qui façonne la personnalité de base et qui permet d'appréhender la culture dans sa totalité. Aussi, Dufrenne prend-il soin de distinguer une sociologie objective qui énonce les faits sociaux, pour les institutions primaires, d'une sociologie psychologique qui vise à décrire l'unité de la culture comme vécue, pour les institutions secondaires. Explication d'un côté, et compréhension de l'autre. Et l'un ne va pas sans l'autre car la compréhension de la culture dans son unité à travers la personnalité de base exige que cette dernière soit saisie d'abord comme l'effet de la causalité du primaire, compris comme un donné auquel elle doit s’adapter. Bref, l'anthropologie culturelle appelle à une collaboration entre les disciplines.

C’est également le point de vue de Lefort qui est cependant moins enthousiaste que Dufrenne sur la portée théorique du concept de personnalité de base, car il récuse la distinction faite par Kardiner entre institutions primaire et secondaire: «L'immense intérêt de la notion de personnalité de base est [...] qu'elle exige le dépassement du réalisme naïf du dualisme individusociété qui l’accompagne» (Lefort, 1951, p. 126).

Le schéma explicatif de Kardiner doit être amendé, précisément parce qu’il a fait une découverte «révolutionnaire », dont il n’a pas tiré tous les bénéfices, en développant l'idée selon laquelle individu et société sont en toute rigueur inséparables (ibid., p. II7). Or Kardiner établirait une juxtaposition entre une tendance qui établit l'interpénétration de l'individuel et du social, et une autre «rétrograde» car elle traite l'un et l'autre comme en action réciproque mais réellement séparés. Cette réserve étant faite, pourvu qu’on ne fasse jouer à la 
personnalité de base aucun rôle causal, Lefort voit dans le travail de Kardiner le premier programme de recherche depuis celui de Mauss qui essaie d'identifier homme total et société totale dans leur immanence.

\subsubsection{G.H.Mead revisité}

Larticle que Paul Kahn consacre à Mead voit l'intérêt de sa «psychologie sociale» dans le fait qu'elle renonce autant à la conception classique de la conscience individuelle close qu'à la conception de la conscience collective substantialisée. Kahn insiste sur l'idée que le développement de la personnalité individuelle est compris à l'intérieur du champ de son expérience, dont on peut saisir l'architecture d'ensemble à partir du concept-clef de symbole. L'important est le mécanisme de symbolisation, ou prise de rôle au moyen duquel l'individu peut prendre les attitudes de la communauté.

Le symbole implique une correspondance d'attitudes entre le Soi et l'Autre. C'est la relation du symbole à cette série de réponses chez l'individu comme chez autrui que Mead appelle symbole signifiant: la signification est incluse à la fois dans l'acte et dans le symbole si bien que conscience réflexive naît en même temps que le symbole et le soi dans l'expérience sociale. Parce qu'il y a symbole, il y a possibilité de prendre envers soi l'attitude que les autres prennent à notre égard. En ce sens, il apparaît enveloppé dans une activité coopérative, un acte social.

Le symbole est donc un processus idéationnel global, dynamique, qui se déroule toujours dans un cadre social, si bien que c'est l'aptitude au symbolisme qui différencie les sociétés humaines. Cela se traduit par une capacité pour l'individu de prendre l'attitude de tous ceux qui sont engagés dans le processus social. Grâce au pouvoir de communiquer les uns avec les autres, et de prendre l'attitude d’autrui, nous portons en nous les systèmes organisés d'attitudes des autres, qui, quand elles sont intériorisées deviennent des motifs de comportement, et une fois extériorisées, des institutions. La pensée de Mead procède d'une intuition juste:

[...]celle de la relativité du symbole par rapport à l'ensemble de l'activité sociale globale. Malheureusement, cette intuition s'accompagne d'une analyse insuffisante de la nature du symbole qui n'est pas clairement distingué du réflexe, du geste, du signe, du signal. (Kahn, 1949, p. I48). 
L'intérêt de lire Mead vient de ce qu'il prouve que l'étude du symbole est une voie idéale pour interpréter la signification des attitudes individuelles dans l'ensemble de la vie sociale.

\subsubsection{Trouver un nouveau régime de causalité pour la sociologie: l'exemple de la réception des ethnologues}

Cette entreprise de «psychologisation» est reliée à tout un ensemble de textes d'ethnologues. Si l'on résume, on pourrait dire que l'ambition est de réussir à combiner empirie et théorie pour montrer comment les différents niveaux de la réalité sociale s'influencent mutuellement, et comment un événement particulier est influencé par des dynamismes globaux. Si les Américains donnent des exemples d'événements et essaient de les expliquer, ils échouent dans leurs tentatives, faute de savoir ce que doit être la causalité. Il faut donc avoir une idée des différents types de causalité qui opèrent à différents niveaux de la réalité sociale, et dissocier les différents régimes de causalité. Avec des variantes dans le vocabulaire utilisé c'est toujours la même critique qui fait figure de leitmotiv, notamment sous la plume de Gurvitch (voir par exemple la critique faite à Mc Iver dans Gurvitch, 1947).

Parmi les contributeurs, ce sont surtout les ethnologues de la Sorbonne qui semblent avoir reçu le message de Gurvitch, et essayé d’appliquer les principes épistémologiques qu'il défend. Prolixes sur cette question de la causalité et de l'inflexion qu'il conviendrait de lui faire subir pour rendre la sociologie opératoire, le détour par les sociétés archaïques, accompli par l’anthropologie culturelle américaine, est pour eux prétexte à une réflexion générale sur la question.

- Une réception de Bastide (...) :

Bastide estime que pour comprendre d'un point de vue sociologique l'évolution des sociétés, il importe d'examiner comment se fait l'intégration à l'ensemble des microprocessus en se plaçant d'un point de vue macrosociologique. D’où l'utilisation de deux types différents de causalités qu'il nomme «interne » et «externe» (Bastide, 1956). 
Selon lui l'anthropologie culturelle américaine utilise une forme de causalité externe quand elle pense le changement social et use de la causalité interne quand elle traite des faits d'acculturation. Le problème est que, la plupart du temps, les Américains en font un mauvais usage: ils passent du psychologisme au sociologisme, et tombent du «système» aux «attitudes». Mais ils auraient surtout tendance à se réfugier dans le psychologisme, en raison de leur tendance au nominalisme et parce qu'ils ont eux-mêmes vécu le changement social comme des rencontres entre civilisations qui ont provoqué des heurts pensés comme une poussière de relations humaines. La solution consiste alors à rétablir au moyen de la double causalité la dimension temporelle et la situation sociale globale où les causes agissent : le milieu interne doit être compris non comme un système formel de relations, mais en dissociant les facteurs.

\section{- (...) et de Cazeneuve :}

Cazeneuve aborde cette problématique quand il se penche sur la question de la «connaissance d’autrui dans les sociétés archaïques» (Cazeneuve, 1958). L’article est tiré de travaux menés sous les auspices du «Groupe de recherches sur la sociologie de la connaissance et la sociologie de la vie morale», créé en 1957, dirigé par Gurvitch, et dont il faisait partie. La connaissance de l'autre est un thème que Cazeneuve considère comme situé à la frontière entre l'ethnologie, la sociologie et la psychologie. Dans ces sociétés, la connaissance d'autrui est problématique, entourée de tabous. Les enfants par exemple qui n’ont pas été soumis au rituel d'initiation sont ainsi considérés comme des animaux. Inversement, des appartenances équivalent à l'individu, une rognure de ses ongles en est le prolongement, fait partie de la personne qui peut de la sorte sortir de la condition humaine.

La question qu'on peut poser est alors de savoir si l'on peut considérer qu'il existe dans chaque société une structure particulière de la personne qui donnerait les cadres de la connaissance d'autrui, ce qui rejoint la notion de personnalité de base, pourtant lacunaire car elle ne permet pas de caractériser ce qui est le moins individuel dans la connaissance d'autrui. Il faudrait un cadre d'analyse plus «souple» susceptible de saisir la réalité vécue et créer une sorte de phénoménologie qui permettrait non pas d'aller de la personne à ses attitudes, comme dans le cas de la théorie de Kardiner, mais «du rapport social, ou plutôt de la "globalité" 
à la personne» (ibid., p. 90). Cette façon de concevoir la connaissance d'autrui permettrait mieux de rendre compte du processus qui dans les sociétés archaïques met un frein à l'individualisation. Par exemple, on comprendrait mieux quelle connaissance des autres suppose le port d'un masque qui est pour les Navajos une identification à un esprit, un an avant la cérémonie destinée à l'invoquer.

\subsubsection{Application: Penser le fait colonial avec les Américains ou la réception de Balandier}

Confronté à des situations de domination coloniale dans un contexte de décolonisation, Balandier tente d’appliquer certains concepts américains aux situations qu'il étudie, compliquées dans les colonies car faites d'émancipations, de reconquêtes, de révoltes, résistances à la métropole etc. C'est lui aussi un argument «culturaliste» qu'il convoque. Les liens de Balandier avec l'anthropologie américaine semblent du reste avoir été suffisamment forts pour qu'il écrive dans un volume collectif publié par les Presses de l'Université de Chicago ${ }^{12}$. Larticle de I95 I qui se penche sur la «situation coloniale», terme que Balandier emprunte à Wirth, exhorte à repenser les rapports entre la métropole et la colonie impliquant des processus complexes d'adaptation et de refus, des points de résistance et des conduites novatrices... Une sociologie américaine des minorités ethniques dominées est conviée pour aider à penser un rapport instrumental complexe entre deux sociétés. Toutefois, si une tradition comme celle de l'anthropologie culturelle américaine aide à penser les changements culturels, elle a le tort à ses yeux de ne jamais envisager la situation coloniale comme une totalité.

La solution consiste alors, en appréciant les apports de la sociologie et de la psychologie sociale aux société colonisées, à considérer celles-ci comme des sociétés globales qui se constituent en «sociétés plurales », c'est-à-dire des groupes plus ou moins conscients de leur existence, opposés les uns aux autres par la couleur et se différenciant par la loi et la coutume. Une minorité européenne s’est superposée à une majorité indigène de civilisation différente en asseyant sa domination sur une supériorité matérielle incontestable, sur un état de droit établi à son avantage 
et sur un système de justification à fondement plus ou moins racial. Balandier prend alors l'exemple des études de Wirth ${ }^{13}$ sur les Noirs vivant dans le Sud.

La bonne façon de corriger les résultats de Wirth consiste à voir le caractère de minorité comme une certaine manière d'être dans la société globale, qui implique essentiellement la relation de dominé à dominant. Montrer en quoi la société colonisée se distingue des autres minorités c'est préciser quelle est sa place dans la société globale. C'est-à-dire, se pencher sur les heurts entre civilisations par le prisme des problèmes raciaux comme le font les Américains, mais en ne les rattachant pas exclusivement à des problèmes culturels.

D’où l'intérêt de la notion de situation de dépendance qu'il importe de caractériser objectivement - en mettant en évidence un certain type de rapports sociaux - mais aussi subjectivement - pour comprendre comment les colonisés éprouvent aussi un sentiment de dépossession spirituelle (Balandier, 1952). Se réclamant de l'autorité de Mead, Balandier rappelle alors que les phénomènes provoqués par la réaction de dépendance varient en fonction de l'arrière-plan socio-culturel. En alliant les ressources de la psycho-sociologie et de l'anthropologie on peut étudier comment la dépendance coloniale est éprouvée par ceux qui la subissent, par une prise de conscience qui agit de différentes façons. Par exemple, quand des institutions fondamentales s'altèrent : en Afrique Centrale naissent des cultes nouveaux, réaction à la christianisation et à la dépossession religieuse qui l’a accompagnée. Ce mouvement impose un regroupement en face du dominant et s'oppose à celui-ci.

Cette prise de conscience des colonisés entraîne une série de comportements conditionnés par la relation de dépendance, qui vont de l'acceptation passive à l'opposition active. L'opposition passive est essentiellement défensive et indirecte : citant I. Hallowell, («Socio-psychological Aspects of Acculturation $»^{14}$ ), il évoque une résistance qui régit certains comportements que les anthropologues ont décrits en termes d’acculturation sous le signe de l’anxiété et du «désajustement

13 II cite "The Problem of minority groups", in The Science of Man in the World crisis, p. 347-372, et The present position of Minorities in the United States.

I4 In Linton (éd.), The science of Man in the World Crisis. 
socio-psychologique ». Il existe aussi des mouvements messianiques qui entretiennent la croyance en un nouvel ordre qui fera revivre l'ancien (voir Balandier dans le volume I4, discutant les travaux de Linton sur l'acculturation).

L'ethnologue est en face d'une réaction qui a des aspects culturels (affirmation et abandon de valeurs), sociologiques (destruction et création de centres de vie sociale) et psychologiques (satisfactions liées au prestige, apparition de racismes), dont les apports conjoints de la sociologie et de l'anthropologie donnent une idée en montrant comment les réactions à cette situation ne peuvent qu'être intériorisées et ne peuvent s'exprimer qu'en étant incorporées. Aussi, une sociologie de la dépendance implique-t-elle «une ré-orientation des recherches, conditionnée par l'élaboration d'un nouvel équipement théorique» (Balandier, 1952, p. 69).

Bref, dans cette utilisation des travaux américains pour rendre compte du vécu des situations sociales, c'est l'argument de la négligence de la dynamique des ensembles sociaux globaux qui est mis en avant.

\section{CONCLUSION}

Ces quelques échantillons de la réception des auteurs américains dans L’Année Sociologique et les Cahiers Internationaux de Sociologie présentent un bilan contradictoire : entre Français et Américains ce n’est ni un rendez-vous manqué, ni une reconnaissance mutuelle. L’intérêt manifesté par la sociologie universitaire française pour certains auteurs américains a surtout servi à lancer une réflexion sur la manière dont l'individu vit sa participation à la vie sociale. Participation dont les Français sont persuadés qu'elle ne prend sens que dès lors qu'elle est rapportée à l'ensemble du milieu social dans lequel elle s'inscrit. Ceci a deux conséquences majeures : il faut rendre la sociologie plus psychologique, et se donner les moyens de restituer la signification vraie des faits «psychologiques» en la rapportant à la totalité du milieu social. C'est là la définition «par défaut» d'une sorte de consensus paradigmatique qu’on observe dans le corpus. 
En somme, contre une tendance constructiviste tenace dans les sciences sociales depuis quelques décennies, qui détourne les sociologues de regarder concrètement les produits des activités qu'ils étudient, nous défendons l'idée qu'il existe quelques invariants dans les ressources cognitives que mobilisent les Français tout au long de leur réception de la sociologie américaine. Cette relative unanimité méritait d'être interrogée plus complètement. Qu’on nomme ces invariants résultats d'un certain «rationalisme» (Berthelot, 2008), postulats ou présupposés théoriques, ils présentent des «régularités autonomes » au sens de Mannheim (Mannheim, 1936), et font figure de référents à partir desquels sont identifiés les «bons» ou les «mauvais » Américains.

\section{BIBLIOGRAPHIE}

BALANDIER Georges, 195I, «La situation coloniale: approche théorique», CIS, vol. II, p.44-79.

-, 1952, «Contribution à une sociologie de la dépendance», CIS, vol. 12, p. 47-69.

-, 1954, «Sociologie de la colonisation et relations entre sociétés globales», CIS, vol. 17., p. |7-31.

-, 1956, «L'expérience de l'ethnologue et le problème de l'explication», CIS, vol. 21, p. II4-127.

BASTIDE Roger, 1956, «La causalité externe et la causalité interne dans l'explication sociologique», CIS, vol. 2I, p.77-99.

BERTHELOT Jean-Michel, 2008, L'Emprise du vrai, Paris, PUF.

BLONDIAUX Loïc, 1991, «Comment rompre avec Durkheim? Jean Stoetzel et la sociologie française de l'après-guerre (1945-1958)», RFS, 32/3), p.4II-44I.

BOURRICAUD François, 1952a, «Quelques remarques sur le concept de "caractère national"», CIS, vol. 12, p. 150-168.

-, 1952b, «Sur la prédominance de l'analyse microsociologique dans la sociologie américaine», CIS, vol. 13, p. 105-121.

-, 1953b, «Essai sur le lien personnel», CIS, vol. 15, p. 64-93. 
CAZENEUVE Jean, 1958, «La connaissance d'autrui dans les sociétés archaïques», CIS, vol. 25, p. 75-99.

CHAPOULIE Jean-Michel, 1991, «La seconde fondation de la sociologie française, les États-Unis et la classe ouvrière», RFS, 32/I), p. 321-364.

CROZIER Michel, 1953, c.r. de V. Jensen Heritage of Conflict, labor relations in the Nonferrous Metals Industry up to 1930, 1950; et de Jones, L'autobiographie de Mama Jones (trad.), 1952, in L'Année Sociologique, 195I, Paris, PUF, p. 47I-472.

DAVY Georges, 1952, c.r. de R. K. Merton, Social theory and social structure: toward the codification of theory and research, 1949, in L'Année Sociologique, 1949-1950, Paris, PUF, p. I5I.

-, 1956, c.r. de Decker Howard, Gillin John, Parsons Talcott et al., For a science of social man: convergence in anthropology, psychology and sociology, 1953, in L'Année Sociologique, 1953-1954, Paris, PUF, p. 172-173.

DUFRENNE Mikel, 1952, «Coup d'œil sur l'anthropologie culturelle américaine», CIS, vol. 12, p. 26-46.

DESMAREZ Pierre, 2004, «Georges Friedmann, médiateur de la sociologie industrielle», in P. Grémion et F. Piotet (dir.), Georges Friedmann. Un sociologue dans le siècle, Paris, CNRS Éditions, p. 103-118.

FRIEDMANN Georges, 1949, «Avant-Propos», c.r. de W.D. Brown, Manager, Men and Morale, 1948; Warner W. L., The social system of the modern factory, 1947; Gillepsie J. J., Free expression in industry, 1948; Saint-Exupéry, Que fautil dire aux hommes? (Le Figaro littéraire), 1948; Romains J., Le Problème numéro un, 1948; Mayo E., The social problems of an industrial civilization, 1945, in L'Année Sociologique, 1940-1948, tome 2, Paris, PUF, p. 76I-765.

-, 1950a, Où va le travail humain?, Paris, Gallimard.

-, 1950b, «L'industrie américaine et le facteur humain», CIS, vol. 8, p. 40-7I.

-, 1952, c.r. de Milton L. Blum, Industrial Psychology and its social foundations, 1949, in L'Année Sociologique, 1949-1950, Paris, PUF, p.436-437.

-, NAVILLE Pierre (éds), 196I, Traité de sociologie du travail, Paris, Armand Colin, (tome 2, 1962). 
GURVITCH Georges, 1947, c.r. de Mc Iver Robert M., Social Causation; et Sorokin Pitrim, Socio-Cultural Causality, Space, Time, CIS, vol. 2, p. 172-I82.

HEILBRON Johan, 1991, «Pionniers par défaut? Les débuts de la recherche au Centre d'études sociologiques», RFS, 32/3), juil.-sept., p. 365-379.

ISAMBERT François-André, ISAMBERT-JAMATI Viviane, 1949, c.r. de G. Friedmann, Problèmes humains du machinisme industriel, in L'Année Sociologique, 1940-1948, tome 2, Paris, PUF, p. 779-782.

ISAMBERT Viviane, 1953, c.r. de William F. Whyte, Pattern for Industrial Peace, in L'Année Sociologique, 195I, Paris, PUF, p. 476-478.

ISAMBERT-JAMATI Viviane, 1956, c.r. de «L'absentéisme de la main-d'œuvre», Revue Française du Travail, 1952; «Les congés de maladie», Bulletin Mensuel de Statistique, 1953; «l'absentéisme dans l'industrie de l'Allemagne orientale», Notes d'Information du gouvernement militaire français, 1953; B. J. Covner, «Management factors affecting absanteeism, Harvard Business Review, sept. 1954; et de D. Hewitt et J. Parfit, «A note on working morale and size of group », Occupational Psychology, janv. 1953, in L'Année Sociologique, 1953-1954, Paris, PUF, p. 447-452.

KAHN Paul, 1949, «Le symbole dans la psychologie sociale de G. H. Mead», CIS, vol. 6, p. 134-149.

LEFORT Claude, 195I, «Notes Critiques sur la méthode de Kardiner», CIS, vol. I0, p. $117-127$.

LOBSTEIN Jacques, 1949, c.r. de Foote W. F. (ed.), Industry and Society, 1946:

- Gardner Burleigh B. , The factory as a social system;

- Lloyd Warner W. et Low J.O., The factory in the community;

- Barnard Chester I., Functions and Pathology of Statutes in formal Organizations;

- Allison Davis, The motivation of the underpriviledged worker;

- Hughe E. C., Race Relations in Industry;

- Whyte W. F., When workers and customers meet,

- Starr M., Rôle of Union Organization;

- Harbison F. H., The basis of industrial conflict; et Shulyer Dean Hoslett, Human factors in management, 1946, in L'Année Sociologique, 1940-1948, tome 2, Paris PUF, p. 797-802. 
MARCEL Jean-Christophe, 200I, «Georges Gurvitch: les raisons d'un succès», CIS, vol. II0, p.97-I19.

-, 2004a, «Une réception de la sociologie américaine en France (1945-1960)», RHSH, n० II, p. 45-68.

-, 2004b, «La réception de Parsons dans la sociologie française de l'aprèsguerre », Durkheimian Studies, vol. 10, n. s., p. 38-55.

-, 2010, Éléments pour une analyse de la réception de la sociologie américaine en France (1945-1959), mémoire pour l'habilitation à diriger des recherches, Université de Paris-Sorbonne, novembre.

MARTIN Olivier et VANNIER Patricia, 2002, «La sociologie française après 1945: places et rôles des méthodes issues de la psychologie », RHSH, n6, p. 95-122.

POLLAK Michael, 1976, «La planification des sciences sociales», ARSS, n², 2-3, juin, p. 105-121.

-, 1988, «La place de Max Weber dans la sociologie française», Droit et société, p. $195-210$.

REYNAUD Jean-Daniel, 1952, c.r. de Current Trends in Industrial Psychology, 1949, in L'Année Sociologique, 1949-1950, Paris, PUF, p. 445-446.

-, 1953, c.r. de E. G. Chambers, Psychology and the Industrial Worker, 195I, «La psychotechnique dans le monde moderne $\gg$. Compte rendu du I $X^{\times}$Congrès international de psychotechnique, 1952, et de E. Ginzberg, S.W. Ginsburg, J.L. Herma., Occupational Choice, an Approach to a General Theory, in L'Année Sociologique, 195I, Paris, PUF, p. 455-458.

-, 1961, «Sociologie et "raison dialectique"», Revue Française de Sociologie, 2/ I, p. 50-66.

STOETZEL Jean, 199| [1946], «L'esprit de la sociologie française contemporaine» dans RFS, 32/3), juil.-sept., p. 443-456.

SCHWEBER Libby, 2002, «Wartime research and the quantification of American sociology. The view from "the American Soldier"), RHSH, 6, p. 65-94.

TOURAINE Alain, 195I, «Classe Sociale et Statut Socio-Économique. (à propos de quelques travaux en langue anglaise) », CIS, vol. II, p. I55-176. 
-, 1952a, c.r. de Friedmann, Où va le travail humain?, 195I, in L'Année Sociologique, 1949-1950, Paris, PUF, p. 419-423.

-, 1952B, «Quelques travaux anglo-saxons récents» c.r. de R. Centers, The Psychology of Social Classes. A Study of Class Consciousness, 1949, J. Hall et D. C. Jones, Social Grading of Occupations, 1950, Ph. Geiss et M. Benney, Social classes and politics, in Greenwitch, 1950, H. J. Eysenck, social Attitude and Social Class V. Sims, Social Class Affiliation of Students in a Southern State University, 1950, F. Finch et J. Hoehn, Measuring socio-economic or cultural Status, a Comparison of Methods, 1951, in L'Année Sociologique, 1949-1950, Paris, PUF, p. 219-225.

-, 1954, «Le Traitement de la société globale dans la sociologie américaine contemporaine», CIS, vol. I6, p. I26-I45.

-, 1956, c.r. de Hariett O. Ronken et Paul R. Lawrence, Administering Change, A case Study of Human Relations in a factory, 1952, in L'Année Sociologique, $1953-$ 1954, Paris, PUF, p. 461-462.

TRÉANTON Jean-René, 1953, c.r. de Delbert C. Miller, et William H. Form, Industrial Sociology, an introduction to the Sociology of Work relations, 1951, in L'Année Sociologique, 1951, Paris, PUF, p. 443-444.

-, 1955, c.r. de Walker C. R., Steeltown. An industrial Case History of the Conflict between Progress and Security, in L'Année Sociologique, 1952, Paris PUF, p. 523-525.

WILLIAMS Richard, 1947, «La psychologie sociale aux États-Unis», CIS, vol. 3, p.68-88. 